\title{
INTRODUCTION \\ Virtual and augmented reality in neurosurgery: a timeline
}

\author{
Walter C. Jean, MD, ${ }^{1}$ Gavin W. Britz, MBBCh, MPH, MBA, ${ }^{2}$ Francesco DiMeco, MD, ${ }^{3}$ \\ Adrian Elmi-Terander, MD, PhD, ${ }^{4}$ and Cameron Mclntyre, $\mathrm{PhD}^{5}$
}

\begin{abstract}
'Division of Neurosurgery, Lehigh Valley Health Network, Allentown, Pennsylvania; ${ }^{2}$ Department of Neurosurgery, Houston Methodist Neurological Institute, Houston, Texas; ${ }^{2}$ Department of Neurosurgery, Fondazione I.R.C.C.S. Istituto Neurologico "C. Besta," Milan, Italy; " ${ }^{4}$ epartment of Neurosurgery, Karolinska University Hospital and Department of Clinical Neuroscience, Karolinska Institute, Stockholm, Sweden; and ${ }^{5}$ Department of Biomedical Engineering, Case Western Reserve University, Cleveland, Ohio
\end{abstract}

$\mathrm{T}$ ECHNOLOGICAL advancement in neurosurgery takes a quantum leap forward every 20 years or so. The microscope entered the neurosurgical operating suite in the late 1950s, and the first commercial CT scans became available in the 1970 s. ${ }^{1,2}$ Mixed reality (MR; i.e., virtual and augmented reality) may represent the next iteration of the leap.

The term "virtual reality" (VR) was coined by the computer scientist turned philosopher Jaron Lanier in the late 1980s. First used for immersive experience in gaming, VR applications in neurosurgery started in the 1990s. But whereas the microscope revolutionized operative visualization, and the CT scans supplanted pneumoencephalograms for making diagnoses, the neurosurgical applications of MR are multifaceted.

As pilots used VR for flight simulation, avoiding damage to airplanes and physical harm to the learner, so too did neurosurgeons start using VR for training, as it allows "do-overs" without jeopardizing patients. What a flight path shares with a surgical approach is the three-dimensionality of complex topography, the terrain for the former and the anatomy of the skull and spine for the latter.

But from that beginning in the acquisition of neurosurgical skill and experience, the application of MR has not only expanded to the assessment of the learner, but also broadened into the realms of surgical planning, operative visualization, and fundamentally changing the nature of neuronavigation. As the studies in this issue demonstrate, the various applications of MR also permeate most of modern-day neurosurgery, from vascular to oncological and spinal procedures.

MR will undoubtedly play a significant role in global neurosurgery in the future, from remote proctoring of complex procedures to the teaching of young neurosurgi- cal trainees around the world. Merged with robotics, a procedure rehearsed, performed, and recorded in VR in one part of the globe may be repeated for a patient in another part of the globe without time zone restrictions. Blended with artificial intelligence, imaging data from thousands of international patients with a specific rare tumor can be collected, turned into virtual models, and tested in VR for various surgical exposures so that the best surgical option can be calculated. Hopefully, as the cost of equipment decreases and internet connectivity increases, the applications of MR will become real for the benefit of patients everywhere.

https://thejns.org/doi/abs/10.3171/2021.5.FOCUS21313

\section{References}

1. Uluç K, Kujoth GC, Başkaya MK. Operating microscopes: past, present, and future. Neurosurg Focus. 2009:27(3):E4.

2. Wijdicks EFM. The first CT scan of the brain: entering the neurologic information age. Neurocrit Care. 2018;28:273275 .

\section{Disclosures}

Dr. Jean reports being a consultant for Surgical Theater LLC. Dr. Elmi-Terander reports being a consultant for Brainlab. Dr. McIntyre reports having ownership in Hologram Consultants and Surgical Information Sciences and being a consultant for Boston Scientific Neuromodulation.

\section{Correspondence}

Walter C. Jean: walter.jean@lvhn.org. 